ARTICLE

\title{
Atomically thin photoanode of InSe/graphene heterostructure
}

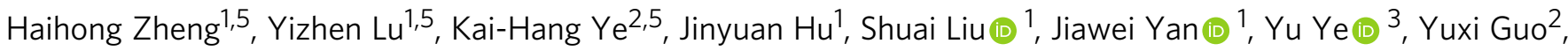 \\ Zhan Lin (1) ${ }^{2}$, Jun Cheng (10 ${ }^{1 凶} \&$ Yang Cao (1) ${ }^{1,4 凶}$
}

Achieving high-efficiency photoelectrochemical water splitting requires a better understanding of ion kinetics, e.g., diffusion, adsorption and reactions, near the photoelectrode's surface. However, with macroscopic three-dimensional electrodes, it is often difficult to disentangle the contributions of surface effects to the total photocurrent from that of various factors in the bulk. Here, we report a photoanode made from a InSe crystal monolayer that is encapsulated with monolayer graphene to ensure high stability. We choose InSe among other photoresponsive two-dimensional (2D) materials because of its unique properties of high mobility and strongly suppressing electron-hole pair recombination. Using the atomically thin electrodes, we obtained a photocurrent with a density $>10 \mathrm{~mA} \mathrm{~cm}^{-2}$ at $1.23 \mathrm{~V}$ versus reversible hydrogen electrode, which is several orders of magnitude greater than other $2 \mathrm{D}$ photoelectrodes. In addition to the outstanding characteristics of InSe, we attribute the enhanced photocurrent to the strong coupling between the hydroxide ions and photogenerated holes near the anode surface. As a result, a persistent current even after illumination ceased was also observed due to the presence of ions trapped holes with suppressed electron-hole recombination. Our results provide atomically thin materials as a platform for investigating ion kinetics at the electrode surface and shed light on developing nextgeneration photoelectrodes with high efficiency.

\footnotetext{
${ }^{1}$ State Key Laboratory of Physical Chemistry of Solid Surfaces, Collaborative Innovation Center of Chemistry for Energy Materials (iChEM), College of Chemistry and Chemical Engineering, Xiamen University, Xiamen 361005, China. ${ }^{2}$ Guangzhou Key Laboratory of Clean Transportation Energy Chemistry, School of Chemical Engineering and Light Industry, Guangdong University of Technology, Guangzhou 510006, China. ${ }^{3}$ State Key Laboratory for Mesoscopic Physics, School of Physics, Peking University, Beijing 100871, China. ${ }^{4}$ Pen-Tung Sah Institute of Micro-Nano Science and Technology, Xiamen University, Xiamen 361005, China. ${ }^{5}$ These authors contributed equally: Haihong Zheng, Yizhen Lu, Kai-Hang Ye. ${ }^{\circledR}$ email: chengjun@xmu.edu.cn; yangcao@xmu.edu.cn
} 
$\mathrm{n}$ photoelectrochemical (PEC) water splitting, the oxygen evolution reaction (OER) is considered to be the rate-limiting step compares to its hydrogen evolution counterpart reaction. This is because OER has four electron transferring processes along with complexities in the coupled ion and photogenerated electron/hole transport mechanism near the photoanode surface ${ }^{1-5}$. As a result, a better understanding of the interactions between ions in the electric double layer and charge carriers in the few atomic layers at the anode surface is critical for developing efficient PEC cells ${ }^{6-8}$. However, despite strong interest, experiments purely investigating the surface process have been proven challenging. The presence of various effects in the bulk electrode, including charge carriers' short diffusion length and bulk recombination, suppress or obscure the efficiency of the surface chemical reactions ${ }^{7,9}$. Nevertheless, strategies to reduce electrode thickness down to tens of nanometers, which is smaller than the hole diffusion length, have been reported with anodes demonstrating enhanced PEC current ${ }^{10,11}$.

Most recently, two-dimensional (2D) crystals have been introduced as effective photoelectrodes materials in PEC reactions with improved photocatalytic properties. Such 2D materials have been demonstrated to have a direct band gap in the visible range, strong light absorption efficiency, and unique light-matter interactions ${ }^{12,13}$. Importantly, the possibility of assembling various $2 \mathrm{D}$ crystals into heterostructures offers another degree of freedom to adjust the band structure of these materials ${ }^{14-17}$. The resulting devices have been demonstrated to have tunable band gaps and elongated photo electron-hole pair lifetimes, which properties are essential for high-efficiency PEC water splitting ${ }^{18-20}$.

a

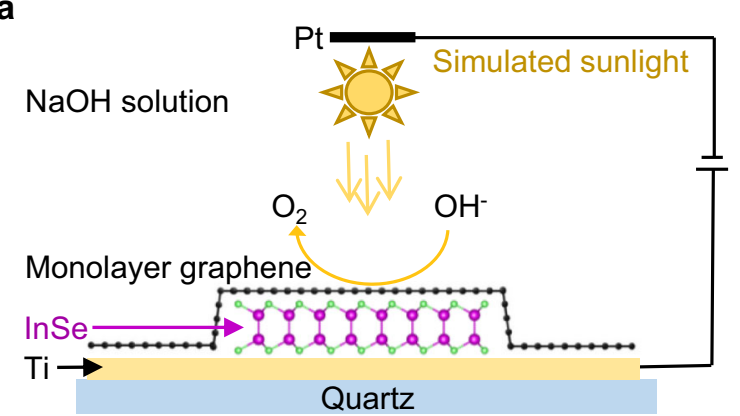

Among all 2D materials, InSe has become a promising candidate for PEC photoanodes due to its excellent charge carrier mobility and its reduced photogenerated electron-hole recombination rate ${ }^{21}$. The valence band edge of InSe is more positive than the oxidative potential of water. Its narrow band gap of $\sim 1.2 \mathrm{eV}$ further facilitates sunlight absorption across broad wavelengths ${ }^{22,23}$. The possibility of reducing InSe crystal thickness down to an atomic-scale makes it a reliable model system for studying surface ion diffusion and adsorption behavior with no influence from its bulk counterparts. However, thin InSe films are sensitive to water and oxygen and are chemically unstable at ambient conditions, which makes using it as an electrochemical electrode challenging 24 .

With this in mind, in this work, we present a new class of PEC anodes made from single-crystal InSe encapsulated by a monolayer graphene, as shown in the schematics in Fig. 1a. The unique feature detailed herein is the atomic-scale thickness of the 2D InSe/graphene (InSe/Gr) photoelectrodes, allowing us to investigate the surface effects on PEC performance. The encapsulation by graphene offers a reliable method to enhance the stability of a monolayer InSe anode over an extended PEC cell operation life. Using the 2D heterostructure-based photoanode, we observed a photocurrent density $>10 \mathrm{~mA} \mathrm{~cm}^{-2}$ at $1.23 \mathrm{~V}$ vs. the reversible hydrogen electrode (RHE). The detailed investigation of both experiments and DFT simulations reveal the importance of hydroxide ion interaction with trapped photogenerated holes near the anode surface. As a result, we also found a persistent current even after illumination ceased when using the thin InSe/graphene photoanode layers, which has not been observed in other $2 \mathrm{D}$ photoelectrodes.

\section{b}

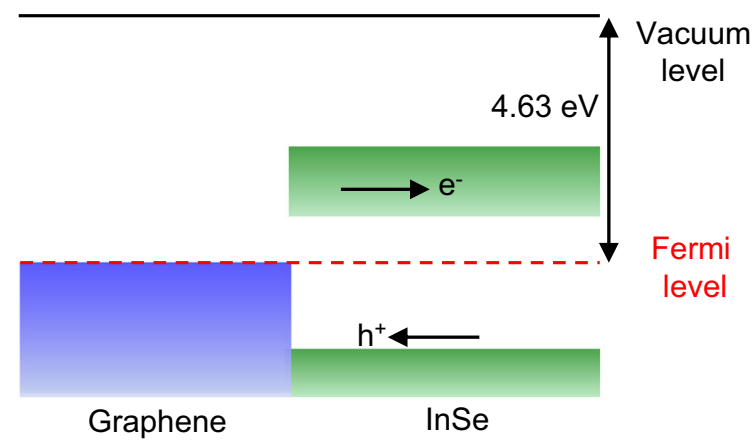

C
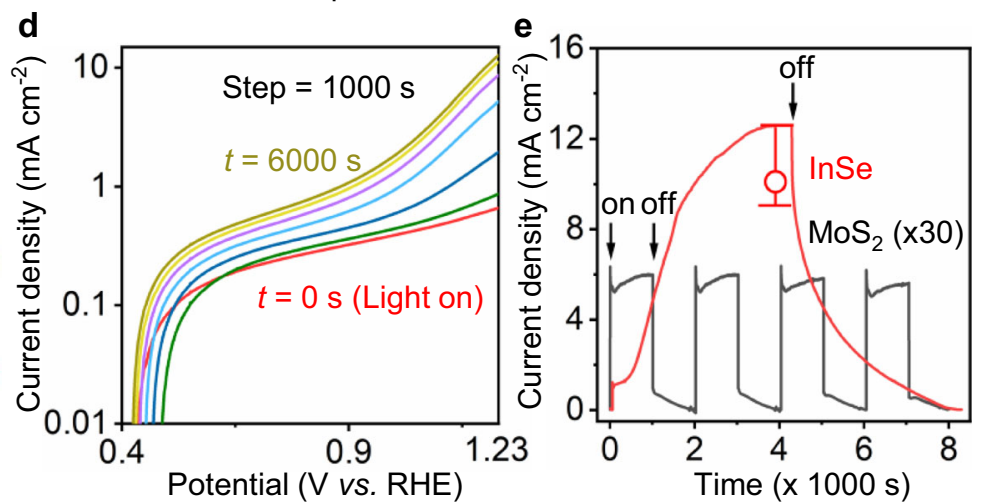

Fig. 1 InSe/Gr anode for PEC measurements. a Schematic of our experiment setup. The illumination used in this report is simulated sunlight: AM $1.5 \mathrm{G}$ one-sun $\left(100 \mathrm{~mW} \mathrm{~cm}^{-2}\right)$. b Band structure of InSe/Gr heterostructure. c DFT simulation of hydroxide species adsorption on InSe/Gr heterostructure surface. Left is the top view. Right is the side view. Red, white, gray, purple, and green balls represent oxygen, hydrogen, carbon, indium and selenium atoms, respectively. The hydroxide species adsorbs on top of the graphene carbon atom. $\mathbf{d}$ Linear sweep voltammetry of a representative monolayer InSe/ Gr device measured at different illumination times. The original linear-scale curves are shown in Supplementary Fig. 5. The Ti electrodes and graphene top layer have little $(<1 \%)$ contribution to the measured total photocurrent (Supplementary Fig. 6). e Photocurrent density of the PEC cell as a function of the time when light was on or off. In this report, all the $J$ - $t$ curves are measured at a potential of $1.23 \mathrm{~V}$ vs. RHE. The blank dot is the saturated maximum current density averaged from 4 samples. The error bar represents the standard deviation error. 


\section{Results}

Fabrication of the InSe/Gr anode. The fabrication of InSe photoelectrodes is described in the "Methods" section. In brief, thin InSe crystals and monolayer graphene were obtained by mechanical exfoliation and then being transferred onto Ti electrodes on a quartz substrate. The unique feature detailed herein is the construction of InSe/graphene van der Waals (vdW) vertical heterostructures (see Supplementary Fig. 1 for an image of a representative InSe/Gr heterostructure and the atomic force microscope (AFM) characterization of the flake thicknesses). In this structure, the chemically less stable InSe crystals were encapsulated by graphene. All the fabrication procedures were performed in a glove box filled with an inert gas (argon) atmosphere to avoid InSe degradation ${ }^{25}$. This encapsulation effectively enabled the high stability of the resulting photoanodes with an InSe thickness down to a monolayer. Atomically thin graphene with optical transparency and high conductivity further facilitates the light absorption and charge carrier transport between InSe and ions in the PEC solution ${ }^{13,19}$.

PEC measurements. The importance of building a InSe/Gr heterostructure can be better understood by analyzing hydroxide ions $\left(\mathrm{OH}^{-}\right)$and photo-generated hole migration near the anode surface using density functional theory (DFT) simulations (Supplementary Methods 'DFT analysis'; Supplementary Figs. 2-4; Supplementary Tables 1-4). As shown in Fig. 1b, the InSe/Gr heterostructure was calculated to have a chemical potential of $-4.6 \mathrm{eV}$ (vs. vacuum), which is well above the OER level of -4.9 $\mathrm{eV}$ at $\mathrm{pH}=13$. The band alignment between InSe and graphene generates a built-in electric field and, in turn, drives holes to the surface of the electrode. In addition, the InSe/Gr heterostructure enables $\mathrm{OH}^{-}$accumulation near the anode surface, with a calculated adsorption energy $\sim-0.74 \mathrm{eV}$ (Fig. 1c). Indeed, a preferential adsorption of hydroxide ions at carbon surfaces has been reported, with a similar adsorption energy on a graphene surface ${ }^{26,27}$. As a result, both negatively charged $\mathrm{OH}^{-}$species and photo-generated holes are enriched near the InSe/Gr anode surface, facilitating their possible interaction.

The current-voltage characteristics of the devices using monolayer InSe were measured in $0.2 \mathrm{M} \mathrm{NaOH}$ solutions and are summarized in Fig. 1d, e. With simulated sunlight illumination, the recorded linear sweep voltammetry (LSV) shows a hydroxide ion oxidation onset potential of $\sim 420 \mathrm{mV}$. The measured photocurrent density $J$ was found to increase as a function of the illumination time $t$, and this yielded a large saturation current density $J_{\max }>10 \mathrm{~mA} \mathrm{~cm}^{-2}$ at $1.23 \mathrm{~V}$ vs. RHE. This value is at least two-fold greater than that of the state-of-art photoanodes, such as $\mathrm{BiVO}_{4}$, which has been demonstrated to have the highest photocurrent density (usually $<5 \mathrm{~mA} \mathrm{~cm}^{-2}$, albeit when decorated with various co-catalyst) among others $^{28,29}$. Moreover, the anode reaction persists even after the illumination ceases, with a typical decay time $t_{\mathrm{D}}$ of $\sim 4000 \mathrm{~s}$ before the observed $J$ decays from $J_{\max }$ to the dark current level. In a typical $J-t$ cycle time of $8000 \mathrm{~s}$, as shown in Fig. 1e, the persistent $J$ contributes $\sim 20 \%$ to the total number of oxidized hydroxide ions and, thus, further enhances the efficiency of the solar energy conversion. There is no obvious degradation of our devices and little variation $(<5 \%)$ in their $J_{\max }$ within a typical lifetime of one month, which confirms the stability of InSe crystals with graphene encapsulation (Supplementary Fig. 7). The importance of the 2D InSe anode to PEC efficiency was further confirmed by the following control experiments. We measured monolayer $\mathrm{MoS}_{2}$ /graphene devices using the same conditions, as shown in Fig. 1e and Supplementary Fig. 8. While there are similarities between InSe and $\mathrm{MoS}_{2}$ (e.g., atomically thin, suitable band diagram for OER and relatively high carrier mobility), the $\mathrm{MoS}_{2}$ shows a fast $J$ photo response with a smaller $J_{\max }$. Such behavior is in consistent with $\mathrm{MoS}_{2}$ anodes previously reported ${ }^{30,31}$.

Hydroxide ion adsorption on the InSe/Gr surface. To gain better insight into the surface effects on the unique performance of the InSe/Gr anode, we first investigated $J_{\max }$ as a function of the $\mathrm{NaOH}$ bulk concentration $C$ (Supplementary Fig. 9). As shown in Fig. 2a, $J_{\max }$ scales proportionally at high $C$ but deviates from the linear dependence and saturates in the limit of small $C$. Such observation is a signature of ion kinetics caused by surface charges, the density of which is independent of the salt concentration ${ }^{32,33}$. Accordingly, the results in Fig. 2a indicate that the $\mathrm{OH}^{-}$concentration near the anode surface $C_{\text {surf }}$ is dominated by both the surface charge density $\rho$ and the bulk concentration $C$. In the low- $C$ region, the constant $J_{\max }$ suggests a constant number of ions adsorbed on surface. The saturation behavior vanishes in the
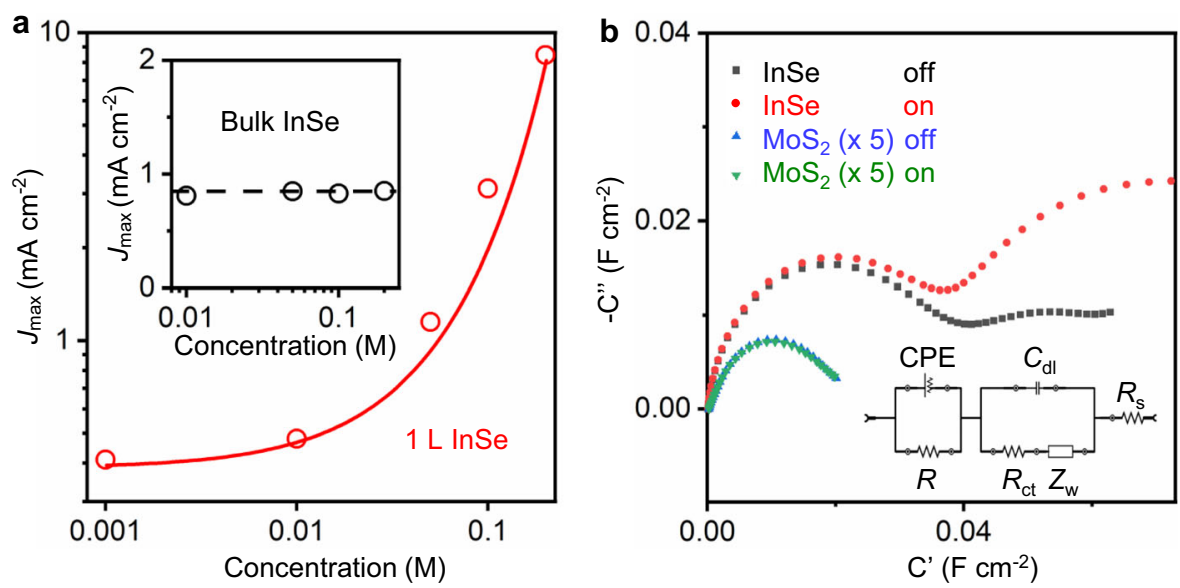

Fig. 2 Hydroxide ion adsorption on the InSe/Gr surface. a $J_{\max }$ of a InSe/Gr monolayer device as a function of the $\mathrm{NaOH}$ concentration. Red dots are the experimental data. The red line is the prediction using Eq. (1) with a surface charge density of $1 \mathrm{mC} \mathrm{m}^{-2}$. The inset is $J_{\max }$ of the bulk (thickness $\sim 1 \mu \mathrm{m}$ ) InSe/Gr device as a function of the $\mathrm{NaOH}$ concentration. The black dots are the experimental data. The black dashed line is to guide the eye. $\mathbf{b}$

Electrochemical impedance spectroscopy of $\mathrm{InSe} / \mathrm{Gr}$ and $\mathrm{MoS}_{2} / \mathrm{Gr}$ monolayer devices when the light is on and off. The inset shows an equivalent electrical circuit of the InSe/Gr anode electrochemical interface. The first semicircle close to origin represents the interface capacitance between the InSe/Gr and the $\mathrm{Ti}$ constant phase element (CPE), and the second semicircle represents the interface capacitance between the InSe/Gr and the PEC solution. For additional details, see Supplementary Methods, 'EIS' section. 
high- $C$ region where the number of ions close to surface is dominated by the bulk concentration. Thus, we quantitatively analyzed $\rho$ :

$$
J_{\max } \propto C_{\text {surf }}=C_{\text {ad }}+C=\frac{\rho}{\mathrm{F} d}+C
$$

where $C_{\text {ad }}$ is the concentration contribution from $\mathrm{OH}^{-}$adsorbed on the surface, $\mathrm{F}$ is the Faraday constant, $d$ is the Stern layer thickness, and $d$ is $\sim 1 \mathrm{~nm}$. Best fitting using Eq. (1) to Fig. 2a yields $\rho \sim 1 \mathrm{mC} \mathrm{m}^{-2}$, which is one order of magnitude smaller than the value recently reported for carbon materials $\left(\sim 10 \mathrm{mC} \mathrm{m}^{-2}\right)^{34}$. We believe that the low surface charge density in our electrodes is due to their clean surface condition. The mechanically exfoliated top graphene contains very few, if any, surface defects ${ }^{35,36}$. Eq. (1) also suggests that at low $C$, hydroxide ions accumulate near the electrode surface with $C_{\text {ad }}$ being significantly greater than $C$, up to 10 times at $C=0.001 \mathrm{M}$ in our case.

It is illustrative to estimate the photo-generated hole concentration $C_{\text {hole }}$ in the InSe anode and to compare that value with $C_{\text {surf }}$, since $J_{\max }$ is expected to be limited by the lower concentration of these two values. After illumination ceases, the measured persistent current comes from $\mathrm{OH}^{-}$oxidation with holes. Thus, we calculated $C_{\text {hole }}=1 / \mathrm{e} \int J \mathrm{~d} t$, where e is the electric charge. The integral is between $t$ when illumination ceases and when $J$ decays to that of the dark state. In monolayer InSe, the above equation yields $C_{\text {hole }}>3 \times$ $10^{19} \mathrm{~cm}^{-2}$ (or $5 \times 10^{5} \mathrm{M}$, with a InSe thickness $\sim 1 \mathrm{~nm}$ ), which is several orders of magnitude greater than $C_{\text {surf. This indicates the }}$ limiting concentration for $J_{\max }$ is $C_{\text {surf }}$, confirming the accuracy of our analysis in Fig. 2a. We note that the estimated $C_{\text {hole }}$ is order of magnitudes greater than that of InSe photoelectric devices measured in vacuum and at ambient conditions ${ }^{21}$. Thus, the surface adsorbed $\mathrm{OH}^{-}$species are hole traps that lead to concentrated holes in the InSe/Gr electrodes. Together with Fig. 2b, where we demonstrate the presence of the hole-enriched surface $\mathrm{OH}^{-}$, our results suggest a strong coupling between the $\mathrm{OH}^{-}$and the photo-generated holes.

The observed surface ion adsorption was further confirmed using electrochemical impedance spectroscopy (EIS). The EIS measurements show that under illumination, the capacitance at the interface of InSe/Gr and the solution is $\sim 2.3$ times greater than that when illumination is off (Fig. 2b and Supplementary Table 5). This result suggests an interaction between the photogenerated holes and $\mathrm{OH}^{-}$species that further enriches the concentration of the latter ${ }^{37}$. This result also matches our DFT analysis of the InSe/Gr band structure. As a reference, the capacitance at the $\mathrm{MoS}_{2} / \mathrm{Gr}$ electrode surface has little dependence on the illumination, with its absolute value being 82 times less than that of InSe/Gr electrodes under illumination (Supplementary Table 5). The smaller capacitance indicates a less favored $\mathrm{OH}^{-}$surface adsorption and is qualitatively in agreement with the lower $J_{\max }$ from $\mathrm{MoS}_{2} / \mathrm{Gr}$ observed in Fig. 1c.

The persistent current after illumination ceases. Having established the $\mathrm{OH}^{-}$-hole interaction governed by the high photocurrent density, we next explore the mechanism behind the persistent $J$ on InSe/Gr anodes after illumination ceases. We measured the photocurrent response in InSe flakes with various thicknesses, which provides information on the surface effects of the InSe/Gr anode. To minimize the contribution of surface charge-induced hydroxide ion accumulation, we used $\mathrm{NaOH}$ solutions having a relatively high concentration of $0.5 \mathrm{M}$. The $J-t$ characteristics in Fig. 3a show that with increasing the InSe flake thickness from a monolayer to $\sim 30$ layers, the decay time $t_{\mathrm{D}}$ of the persistent $J$ gradually decreases by one order of magnitude. For bulk InSe $(>100 \mathrm{~nm})$, as shown in Fig. $3 \mathrm{c}$, such a slow photocurrent response was not detectable (within our measurement accuracy, $<200 \mathrm{~ms}$ ). The corresponding $J_{\max }$ is approximately one order of magnitude less than that of the monolayer, although $C_{\text {surf }}$ is dominated by $C$ and is expected to be similar for both monolayer and thick InSe flakes.

The decay of the photocurrent can be described by an exponential function:

$$
J(t)=J_{0}+A \exp (-k t)
$$

where $J_{0}$ and $A$ are constants. $k$ is a time constant and describes the rate of current decay. The best fit of our results using Eq. (2) demonstrated a dramatic increase in $k$ when increasing the InSe crystal thickness (as shown in Fig. 3b). This indicates that the recombination of photogenerated electron-hole pairs in monolayer InSe was strongly suppressed due to the interaction between the ions and trapped holes. Similar suppressive behavior was also observed in a previous report ${ }^{21}$. However, in thick InSe, the total number of photo-generated holes is expected to be greater than that of the monolayers due to its reduced transparency and smaller bandgap energy ${ }^{38}$. The direct-to-indirect band gap transition from bulk to monolayer InSe further facilitates the photoelectric transition for thick crystals $^{39}$. However, Fig. 3 suggests a less efficient PEC performance for the bulk InSe anode with a fewer number of photogenerated holes reacting with $\mathrm{OH}^{-}$ after illumination ceased. We attribute the large $k$ and smaller $J_{\max }$ observed in thick InSe to the bulk recombination of photogenerated electrons and holes, and thus, the surface hydroxide groups trapped holes have less effect.
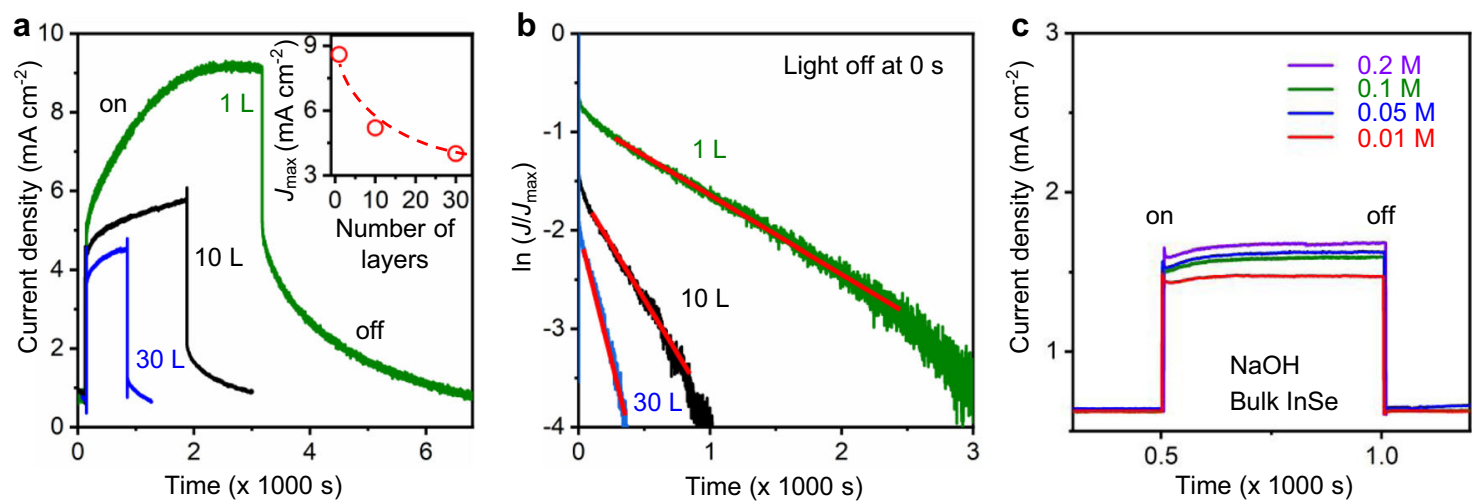

Fig. 3 Persistent photocurrent density in the InSe/Gr photoanode. a Current density as a function of illumination time for InSe with various thicknesses. Inset shows the observed $J_{\max }$ as a function of the InSe thickness. b Photocurrent decay for InSe with various thicknesses. The time point at which the light ceased is defined as $t=0$ for each sample. The red lines are the best fit using Eq. (2). c Temporal dependence of the bulk InSe/Gr anode with various $\mathrm{NaOH}$ concentrations. 


\section{Discussion}

We note that the InSe/Gr heterostructure is critical to the observed high photocurrent. To demonstrate that, we performed a series of control experiments. First, although the photothermal effect has been reported to have a profound influence on the prolonged photocurrent ${ }^{40,41}$, we excluded its influence on the observed slow current response using few-layer InSe (e.g., Fig. 1e, 3). As demonstrated in the Supplementary Methods 'Photothermal effects' and Supplementary Fig. 10, we used Raman spectroscopy and found that both monolayer and thick InSe/Gr heterostructures show a similar temperature variation trend during illumination, regardless of their different current decay time constant, as shown in Fig. 3b.

Second, various interactions between the substrate and 2D heterostructures, including lattice mismatch ${ }^{42,43}$, hybridization ${ }^{44}$ and band alignment ${ }^{45}$, have been reported. In our case, the results were acquired from $\mathrm{InSe} / \mathrm{Gr}$ on a $\mathrm{Ti}$ substrate supported by quartz (see Fig. 1a and "Methods" section for sample schematic). To exclude the influence of the substrate on the measured PEC performance, we investigated the InSe/Gr heterostructure on various substrates, including $\mathrm{Ti}, \mathrm{Cu}, \mathrm{Au}$, and graphite, with distinct crystallinity and lattice constants, as shown in Fig. 1e and Supplementary Fig. 11. Both the photocurrent value and its evolution with time are comparable for all devices under the same experimental conditions, indicating that the substrate has a minor effect on the PEC performance.

Combined with DFT simulation, we attribute the high photocurrent density and persistent current after illumination to the interaction between the hydroxide ions and the photogenerated holes on the anode surface. According to the band alignment and partial density of states (PDOS) of InSe/Gr and $\mathrm{MoS}_{2} / \mathrm{Gr}$ heterojunctions (as shown in Supplementary Methods 'DFT analysis' and Supplementary Fig. 3), the lower energy of the InSe valence band facilitates the migration of photogenerated holes from the InSe to the graphene surface ${ }^{46}$.

Moreover, we also investigated the total energy difference $(\Delta E)$ before and after the reaction:

$$
\mathrm{OH}^{-}+\mathrm{h}^{+} \rightarrow \mathrm{OH}^{*}
$$

which is usually considered to be the initial step of the OER reaction. The concentration of bound hydroxide ions at the InSe/ Gr surface can be estimated as $C_{\text {surf }}$ InSe $\approx C \exp \left(-\Delta E^{\mathrm{InSe}} / k_{\mathrm{B}} T\right)$, where $k_{\mathrm{B}}$ is the Boltzmann constant. An analogous expression applies to the $\mathrm{MoS}_{2} / \mathrm{Gr}$ surface as well. Since $I_{\max }$ is proportional to $C_{\text {surf }}$, it is not unreasonable to assume that $J_{\max }$ InSe $/ J_{\max } \mathrm{MoS} 2=$ $C_{\text {surf }}$ InSe $/ C_{\text {surf }}$ MoS2. Using our results in Fig. 1e and the above expressions, we find the absolute value of $\Delta E^{\mathrm{MoS} 2}-\Delta E^{\mathrm{InSe}} \approx 0.1$ $\mathrm{eV}$. The lower $\Delta E$ for InSe/Gr compared to that of $\mathrm{MoS}_{2} / \mathrm{Gr}$ is supported by our DFT calculations that found $\Delta E^{\mathrm{MoS} 2}-$ $\Delta E^{\mathrm{InSe}} \approx 0.117 \mathrm{eV}$ (Supplementary Methods 'DFT analysis' and Supplementary Table 4). The deviation between the experiments and theory is perhaps not a surprise due to the complexity of the hydration shells of the ions in solution compare to that of ions in vacuum, which is assumed in simulations. These results indicate that the reaction of hydroxide ions with holes has a lower activation energy barrier on the surface of InSe/Gr, resulting in ion accumulation and, thus, a greater current density. For the same reason, the holes trapped by hydroxide ions on the InSe/Gr electrode surface suppress the surface electron-hole recombination, leading to the persistent current after illumination.

In conclusion, we provide insight into the strong coupling between surface adsorbed ions and photo-generated holes, which is critical for achieving highly efficient PEC water splitting. Our results demonstrate a versatile method of stabilizing chemically sensitive electrode materials and emphasize the importance of choosing specific $2 \mathrm{D}$ electrodes to tune ion kinetics near the electrode surface. Due to the large family of novel $2 \mathrm{D}$ materials and the compatibility of van der Waals heterostructures based on them ${ }^{15}$, these atomically thin materials with various surface properties can be used as a model system to study the mechanism of surface processes/reactions or can be integrated with conventional 3D electrodes to modify the electrode's surface and achieve better electrochemical performance.

\section{Methods}

Photoanode fabrication. To avoid the degradation of the ultrathin InSe during the following measurements, exfoliation and encapsulation of the InSe photoanodes were carried out in an inert atmosphere provided by a glovebox $\left(\mathrm{H}_{2} \mathrm{O}\right.$ and $\mathrm{O}_{2}$ less than $0.1 \mathrm{ppm}$ ). Schematic diagrams of the InSe/Gr anode fabrication process are shown in Supplementary Fig. 12. First, InSe flakes were mechanically exfoliated onto a thin polypropylene carbonate (PPC) film. Then, a monolayer InSe crystal was identified using an optical microscope and was lifted with monolayer graphene (Gr) attached to polymethyl methacrylate (PMMA) membranes using a dry peel transfer technique ${ }^{21,25}$. The resulting Gr-InSe stack was transferred onto a 30-nm Ti electrode with a quartz substrate prepatterned using photolithography and ebeam evaporation. The obtained heterostructures were heated at $70{ }^{\circ} \mathrm{C}$ for $1 \mathrm{~h}$ and then the PMMA was dissolved in acetone and isopropanol. Since the single top graphene layer is impermeable to all gas and liquid molecules ${ }^{47}$, such encapsulation can protect the ultrathin InSe during the following PEC measurements and prevent surface contamination. Finally, the sample was removed from the glovebox to verify the InSe thickness using atomic force microscope. Similar fabrication procedures were used for the $\mathrm{MoS}_{2} / \mathrm{Gr}$ anodes.

PEC measurements. All the measurements were carried out on an electrochemical workstation (CHI 750E, Chenhua, Shanghai) using a custom-made, threecompartment photoelectrochemical cell with a $\mathrm{Hg} / \mathrm{HgO}$ reference electrode and a $\mathrm{Pt}$ mesh counter electrode, and the electrolyte was an $\mathrm{NaOH}$ solution. The measured potentials were converted to the RHE scale using the equation $E_{\mathrm{RHE}}=E_{\mathrm{Hg} / \mathrm{HgO}}+$ $0.059 \times \mathrm{pH}+0.098$. Light illumination was applied at AM $1.5 \mathrm{G}\left(100 \mathrm{~mW} \mathrm{~cm}^{-2}\right)$ using a class AAA solar simulator (XES-40S3-TT, San-Ei Electric, Japan). Linear sweep voltammetry curves were performed at scan rate of $5 \mathrm{mV} \mathrm{s}^{-1}$. Electrochemical impedance spectroscopy (EIS) was measured by using a sinusoidal signal with amplitude of $5 \mathrm{mV}$ over a frequency range of $0.1-10 \mathrm{kHz}$, and the scanning potential was $1.23 \mathrm{~V}$ vs. RHE.

\section{Data availability.}

The data that support the findings of this study are available from the corresponding author upon reasonable request.

Received: 18 June 2020; Accepted: 25 November 2020; Published online: 04 January 2021

\section{References}

1. Lu, F., Zhou, M., Zhou, Y. \& Zeng, X. First-row transition metal based catalysts for the oxygen evolution reaction under alkaline conditions: basic principles and recent advances. Small 13, 1701931:1-18 (2017).

2. Meng, L. et al. A plasma-triggered $\mathrm{O}-\mathrm{S}$ bond and $\mathrm{P}-\mathrm{N}$ junction near the surface of a $\mathrm{SnS}_{2}$ nanosheet array to enable efficient solar water oxidation. Angew. Chem. Int. Ed. 58, 16668-16675 (2019).

3. Meng, L. et al. Simultaneous manipulation of O-doping and metal vacancy in atomically thin $\mathrm{Zn}_{10} \mathrm{In}_{16} \mathrm{~S}_{34}$ nanosheet arrays toward improved photoelectrochemical performance. Angew. Chem. Int. Ed. 57, 16882-16887 (2018).

4. Takata, T. et al. Photocatalytic water splitting with a quantum efficiency of almost unity. Nature 581, 411-414 (2020).

5. Wang, H. et al. Highly active deficient ternary sulfide photoanode for photoelectrochemical water splitting. Nat. Commun. 11, 3078:1-11 (2020)

6. Wang, W., Xu, M., Xu, X., Zhou, W. \& Shao, Z. Perovskite oxide based electrodes for high-performance photoelectrochemical water splitting. Angew. Chem. Int. Ed. 59, 136-152 (2020).

7. Ding, C., Shi, J., Wang, Z. \& Li, C. Photoelectrocatalytic water splitting: significance of cocatalysts, electrolyte, and interfaces. ACS Catal. 7, 675-688 (2017).

8. Jiang, C., Moniz, S. J. A., Wang, A., Zhang, T. \& Tang, J. Photoelectrochemical devices for solar water splitting-materials and challenges. Chem. Soc. Rev. 46, 4645-4660 (2017).

9. Wang, Q. \& Domen, K. Particulate photocatalysts for light-driven water splitting: mechanisms, challenges, and design strategies. Chem. Rev. 120, 919-985 (2020). 
10. Shi, X. et al. Efficient photoelectrochemical hydrogen production from bismuth vanadate-decorated tungsten trioxide helix nanostructures. Nat. Commun. 5, 4775:1-8 (2014).

11. Qiu, Y. et al. Efficient photoelectrochemical water splitting with ultrathin films of hematite on three-dimensional nanophotonic structures. Nano Lett. 14, 2123-2129 (2014).

12. Zhang, $\mathrm{X}$. et al. Computational screening of $2 \mathrm{D}$ materials and rational design of heterojunctions for water splitting photocatalysts. Small Methods 2, 1700359:1-9 (2018).

13. Faraji, M. et al. Two-dimensional materials in semiconductor photoelectrocatalytic systems for water splitting. Energy Environ. Sci. 12, 59-95 (2019).

14. Novoselov, K. S., Mishchenko, A., Carvalho, A. \& Castro Neto, A. H. 2D materials and van der Waals heterostructures. Science 353, aac9439:1-11 (2016).

15. Liu, Y. et al. Van der Waals heterostructures and devices. Nat. Rev. Mater. 1, 16042:1-17 (2016).

16. Tartakovskii, A. Excitons in 2D heterostructures. Nat. Rev. Phys. 2, 8-9 (2020).

17. Lyu, J., Pei, J., Guo, Y., Gong, J. \& Li, H. A new opportunity for 2D van der Waals heterostructures: making steep-slope transistors. Adv. Mater. 32, 1906000:1-8 (2020).

18. $\mathrm{Si}$, K. et al. A two-dimensional $\mathrm{MoS}_{2} / \mathrm{WSe}_{2}$ van der Waals heterostructure for enhanced photoelectric performance. Appl. Surf. Sci. 507, 145082:1-11 (2020).

19. Li, C. et al. Engineering graphene and TMDs based van der Waals heterostructures for photovoltaic and photoelectrochemical solar energy conversion. Chem. Soc. Rev. 47, 4981-5037 (2018).

20. Shi, J. et al. Temperature-Mediated selective growth of $\mathrm{MoS}_{2} / \mathrm{WS}_{2}$ and $\mathrm{WS}_{2} /$ $\mathrm{MoS}_{2}$ vertical stacks on Au foils for direct photocatalytic applications. Adv. Mater. 28, 10664-10672 (2016).

21. Bandurin, D. A. et al. High electron mobility, quantum Hall effect and anomalous optical response in atomically thin InSe. Nat. Nanotechnol. 12, 223-227 (2017).

22. Li, Z. et al. High-performance photo-electrochemical photodetector based on liquid-exfoliated few-layered InSe nanosheets with enhanced stability. $A d v$. Funct. Mater. 28, 1705237:1-7 (2018).

23. Shen, T., Ren, J. C., Liu, X., Li, S. \& Liu, W. Van der Waals stacking induced transition from schottky to ohmic contacts: 2D metals on multilayer InSe. J. Am. Chem. Soc. 141, 3110-3115 (2019).

24. Ho, P. H. et al. High-mobility InSe transistors: the role of surface oxides. ACS Nano 11, 7362-7370 (2017).

25. Cao, Y. et al. Quality heterostructures from two-dimensional crystals unstable in air by their assembly in inert atmosphere. Nano Lett. 15, 4914-4921 (2015).

26. Grosjean, B. et al. Chemisorption of hydroxide on 2D materials from DFT calculations: graphene versus hexagonal boron nitride. J. Phys. Chem. Lett. 7, 4695-4700 (2016).

27. Grosjean, B., Bocquet, M. L. \& Vuilleumier, R. Versatile electrification of twodimensional nanomaterials in water. Nat. Commun. 10, 1656:1-8 (2019).

28. Ye, K. H. et al. Carbon quantum dots as a visible light sensitizer to significantly increase the solar water splitting performance of bismuth vanadate photoanodes. Energy Environ. Sci. 10, 772-779 (2017).

29. Jian, J. et al. Embedding laser generated nanocrystals in $\mathrm{BiVO}_{4}$ photoanode for efficient photoelectrochemical water splitting. Nat. Commun. 10, 2609:1-9 (2019).

30. Wu, C. et al. A critical review on enhancement of photocatalytic hydrogen production by molybdenum disulfide: from growth to interfacial activities. Small 15, 1900578:1-25 (2019).

31. Pesci, F. M. et al. $\mathrm{MoS}_{2} / \mathrm{WS}_{2}$ heterojunction for photoelectrochemical water oxidation. ACS Catal. 7, 4990-4998 (2017).

32. Siria, A. et al. Giant osmotic energy conversion measured in a single transmembrane boron nitride nanotube. Nature 494, 455-458 (2013).

33. Feng, J. et al. Single-layer $\mathrm{MoS}_{2}$ nanopores as nanopower generators. Nature 536, 197-200 (2016)

34. Secchi, E., Nigues, A., Jubin, L., Siria, A. \& Bocquet, L. Scaling behavior for ionic transport and its fluctuations in individual carbon nanotubes. Phys. Rev. Lett. 116, 154501:1-5 (2016).

35. Bolotin, K. I. et al. Ultrahigh electron mobility in suspended graphene. Solid State Commun. 146, 351-355 (2008).

36. Mayorov, A. S. et al. Micrometer-scale ballistic transport in encapsulated graphene at room temperature. Nano Lett. 11, 2396-2399 (2011)

37. Ji, H. et al. Capacitance of carbon-based electrical double-layer capacitors. Nat. Commun. 5, 3317:1-7 (2014).
38. Sun, Y. et al. InSe: a two-dimensional material with strong interlayer coupling. Nanoscale 10, 7991-7998 (2018).

39. Mudd, G. W. et al. Tuning the bandgap of exfoliated inse nanosheets by quantum confinement. Adv. Mater. 25, 5714-5718 (2013).

40. Zhan, C. et al. Disentangling charge carrier from photothermal effects in plasmonic metal nanostructures. Nat. Commun. 10, 2671:1-8 (2019).

41. Yang, $H$. et al. Tunable wavelength enhanced photoelectrochemical cells from surface plasmon resonance. J. Am. Chem. Soc. 138, 16204-16207 (2016).

42. Bakkers, E. et al. Epitaxial growth of InP nanowires on germanium. Nat. Mater. 3, 769-773 (2004)

43. Liu, Y., Huang, Y. \& Duan, X. Van der Waals integration before and beyond two-dimensional materials. Nature 567, 323-333 (2019).

44. Kistanov, A. A., Cai, Y., Zhou, K., Dmitriev, S. V. \& Zhang, Y.-W. Effects of graphene/BN encapsulation, surface functionalization and molecular adsorption on the electronic properties of layered InSe: a first-principles study. Phys. Chem. Chem. Phys. 20, 12939-12947 (2018).

45. Bae, S. et al. Integration of bulk materials with two-dimensional materials for physical coupling and applications. Nat. Mater. 18, 550-560 (2019).

46. Moniz, S. J. A., Shevlin, S. A., Martin, D. J., Guo, Z.-X. \& Tang, J. W. Visiblelight driven heterojunction photocatalysts for water splitting - a critical review. Energy Environ. Sci. 8, 731-759 (2015).

47. Sun, P. Z. et al. Limits on gas impermeability of graphene. Nature 579, 229-232 (2020).

\section{Acknowledgements}

The experimental work was supported by the National Key R\&D Program of China (2018YFA0306900 and 2018YFA0209500), the National Natural Science Foundation of China (21872114), and China Postdoctoral Science Foundation (2020M682616).

\section{Author contributions}

H.H.Z., Y.Z.L., and K.H.Y. performed the experiments and data analysis. J.Y.H. and J.C. contributed to DFT simulations. S.L. and J.W.Y. contributed to EIS analysis. Y.Y. helped with device fabrication. G.Y.X. and L.Z. helped with the PEC measurements. Y.C. supervised the research work. The manuscript was written by Y.C. with helps from Y.Z. L., K.H.Y., and H.H.Z. All the authors contributed to discussions.

\section{Competing interests}

The authors declare no competing interests.

\section{Additional information}

Supplementary information is available for this paper at https://doi.org/10.1038/s41467020-20341-7.

Correspondence and requests for materials should be addressed to J.C. or Y.C.

Peer review information Nature Communications thanks W X Zhang, and the other, anonymous, reviewer(s) for their contribution to the peer review of this work. Peer reviewer reports are available.

Reprints and permission information is available at http://www.nature.com/reprints

Publisher's note Springer Nature remains neutral with regard to jurisdictional claims in published maps and institutional affiliations.

Open Access This article is licensed under a Creative Commons Attribution 4.0 International License, which permits use, sharing, adaptation, distribution and reproduction in any medium or format, as long as you give appropriate credit to the original author(s) and the source, provide a link to the Creative Commons license, and indicate if changes were made. The images or other third party material in this article are included in the article's Creative Commons license, unless indicated otherwise in a credit line to the material. If material is not included in the article's Creative Commons license and your intended use is not permitted by statutory regulation or exceeds the permitted use, you will need to obtain permission directly from the copyright holder. To view a copy of this license, visit http://creativecommons.org/ licenses/by/4.0/.

(C) The Author(s) 2021 